\title{
Fabrication of Cellulose Acetate/Cellulose-HA Composite Films for Bone Fixation
}

\section{FATIMA Nisar ${ }^{a}$, USMAN bin Khalid ${ }^{b}$, MUHAMMAD Aftab Akramc, SOFIA Javed ${ }^{\mathrm{d}}$ and MOHAMMAD Mujahid ${ }^{\mathrm{e}}$}

\author{
School of Chemicals and Materials Engineering, NUST, Islamabad, Pakistan \\ afatima_me06@scme.nust.edu.pk, busmanbinkhalid992@gmail.com, \\ caftabakram@scme.nust.edu.pk, ${ }^{d}$ sofia.javed@scme.nust.edu.pk, ${ }^{e} \mathrm{~m} . \mathrm{mujahid@scme.nust.edu.pk}$
}

\begin{abstract}
Keywords: Micro Cellulose, Cellulose Acetate, Membranes, Bone, Hydroxyapatite, Biomedical Material, Bone, X-Ray Diffraction, Scanning Electron Microscopy, Simulated Body Fluid, FTIR.
\end{abstract}

\begin{abstract}
Bone is a rigid and constantly remodeling organ, a type of tissue which provides support and protects organs in the body, and together they form the skeleton. Sometimes due to trauma or injury, the damage requires orthopedic surgery. Materials generally used for implants bear tissue rejection and produce toxins on degradation. The objective is to synthesize a biocompatible film which mimics the properties of natural bone that can be used for bone replacements. Hydroxyapatite (HA) and Cellulose are used as a reinforcement and Cellulose Acetate as a matrix. The experimental procedure is divided into two major steps; extraction of cellulose microfibers (CMF) from raw cotton followed by dispersion of cellulose and HA in cellulose acetate then casting membranes of the composite. The composite film is successfully fabricated through biometric route. The porous and flexible films obtained allow osteoconductivity and Hydroxyapatite growth in simulated body fluid. X-Ray diffraction; scanning electron microscopy and Fourier transform infrared spectroscopy are used to characterize the films.
\end{abstract}

\section{Introduction}

Bones, being an integral part of the human body, perform numerous functions and are responsible for the structural framework of our body [1]. They start developing at a very early stage by the bone apposition process and with the passage of time this process slows down which leads to weakening of bones [2]. Damage may also result due to injuries caused by accidents. Synthetic materials are required to compensate for this deterioration of bones which are used as implants but most of these are composed of metals and other materials which are not readily accepted by the body and result in tissue rejection. Therefore, an alternative biocompatible material is required with similar structural properties.

A material is required which is flexible and can be made into a form which can replace the bone as an implant. The process selection and the synthesis of that material is the main focus of this project. First the components of bone are analyzed and then material selection is done based on the composition of the actual bone. These are hydroxyapatite, cellulose and cellulose acetate $[3,4]$.

Hydroxyapatite is a calcium phosphate which is already found in the bone and makes up most of the bone $[5,6]$. It is a ceramic with hexagonal crystal structure. It is a bioactive and biocompatible material [7-9]. Cellulose from plant is the other material because it is also biocompatible and abundant. It is used to mimic collagen which is present in the bones as cellulose is the structural material in plants just as collagen is the structural material in bones of humans and animals $[5,10]$. First phase of the project was the extraction of cellulose from cotton fibers as cotton has lignin and hemicellulose in it. This was done by the acid hydrolysis method and the concentration of acid was varied to obtain microfibers of varying lengths $[10,11]$.

In the next phase two methods were selected for the formation of the composite of hydroxyapatite which was used as obtained. The first method was to deposit hydroxyapatite on the cellulose fibers in a $1.5 \mathrm{r}$-sbf using the method similar to that which happens in body [12, 13]. The second method was a simple solution casting method in which cellulose acetate $[14,15]$ was used 
as a matrix in which hydroxyapatite and cellulose were dispersed as reinforcements and then the membrane developed was analyzed [16].

The former process follows a biometric route and allows bone regeneration due to osteoconductivity through porous membranes. This being a unique approach would open the doors for further research on self-healing bones. In future the membranes can be stacked together to enhance their properties or even rolled to obtain flexible rods with good mechanical properties.

\section{Experimental Procedure}

Extraction of Cellulose. The cotton is first washed then sourced and bleached in 20 wt. \% sodium hydroxide $\mathrm{NaOH}$ for 2 hours at $70^{\circ} \mathrm{C}$. This swells the cotton and dissolves impurities such as lignin, hemicellulose and see, etc. After soaking and stirring in $\mathrm{NaOH}$ cotton is washed by distilled water to neutralize the $\mathrm{pH}$. Thereafter the washed cotton is torn into short fiber and stirred for further 2 hours at $70^{\circ} \mathrm{C}$ in sulfuric acid $\mathrm{H}_{2} \mathrm{SO}_{4}$. This step is basically to reduce the chain length and degree of polymerization of cellulose as acids interacts with the amorphous region in cellulose and breaks intermolecular bonds $[5,10]$. The acidic suspension of cellulose microfibers is then left to settle and acid is decanted, distilled water is used to wash and neutralize the acidic microfibers. The fibers are obtained by filtration and dried in oven at $60^{\circ} \mathrm{C}$ for an hour. The dried product is then treated mechanically, that is pestle and mortar is used to crush fibers to reduce their size further and also to de-agglomerate the fibers $[11,17]$. The long cellulose molecules are now converted to short micro fibers which can be used to enhance the strength as well as provide flexibility.

Simulated Body Fluid. It can be used for not only measurement of bioactivity of artificial materials in vitro, but also for apatite coatings on various materials through biomimetic route in controlled environment. In our case simulated body fluid is used for precipitation of calcium phosphate in the form of hydroxyapatite (HA) on pre-treated micro cellulose [18].

Standard steps to prepare revised simulated body fluid (R-SBF) were followed. Firstly, apparatus was washed with HCL (to sterilize) and then by deionized or double distilled water, thereafter while stirring, each chemical was added to $1000 \mathrm{ml}$ deionized water at $36.5^{\circ} \mathrm{C}$ in the quantity as mentioned in the table 1 till order\#8 and reagent 9 added slowly in quantity less than $1 \mathrm{~g}$ to avoid increase in $\mathrm{pH}$ locally. After adjusting $\mathrm{pH}$ to 7.25 at a temperature of $36.5^{\circ} \mathrm{C}$ the fluid was left for $1-2$ days to be stable [19].

Table 1 Reagents used to prepare simulated body fluid

\begin{tabular}{|c|c|c|c|}
\hline Order & Reagent & SBF in $1000 \mathrm{ml}$ & $1.5 \mathrm{SBF}$ in $1000 \mathrm{ml}$ \\
\hline 1 & Ultrapure water & & \\
\hline 2 & $\mathrm{NaCl}$ & $7.996 \mathrm{~g}$ & $11.994 \mathrm{~g}$ \\
\hline 3 & $\mathrm{NaHCO}_{3}$ & $0.350 \mathrm{~g}$ & $0.525 \mathrm{~g}$ \\
\hline 4 & $\mathrm{KCl}$ & $0.224 \mathrm{~g}$ & $0.336 \mathrm{~g}$ \\
\hline 5 & $\mathrm{~K}_{2} \mathrm{HPO}_{4} \cdot 3 \mathrm{H}_{2} \mathrm{O}$ & $0.228 \mathrm{~g}$ & $0.342 \mathrm{~g}$ \\
\hline 6 & $\mathrm{MgCl}_{2} \cdot 6 \mathrm{H}_{2} \mathrm{O}$ & $0.305 \mathrm{~g}$ & $0.458 \mathrm{~g}$ \\
\hline 7 & $1 \mathrm{kmol}^{3} \mathrm{HCl}$ & $40 \mathrm{~cm}^{3}$ & $60 \mathrm{~cm}^{3}$ \\
\hline 8 & $\mathrm{CaCl}_{2}$ & $0.278 \mathrm{~g}$ & $0.417 \mathrm{~g}$ \\
\hline 9 & $\mathrm{Na}_{2} \mathrm{SO}_{4}$ & $0.071 \mathrm{~g}$ & $0.107 \mathrm{~g}$ \\
\hline 10 & $\left(\mathrm{CH}_{2} \mathrm{OH}\right)_{3} \mathrm{CNH}_{2}$ & $6.057 \mathrm{~g}$ & $9.86 \mathrm{~g}$ \\
\hline
\end{tabular}

Composite Formation. 50ml of SBF prepared as mentioned previously is used with a $\mathrm{pH}$ adjusted around 7.5 with Tris(hydroxymethyl) aminomethane, at a temperature of $36^{\circ} \mathrm{C}$. CNC suspension is made $(\sim 0.34 \mathrm{mmol} / \mathrm{g})$ and placed in ultrasonic bath for $15 \mathrm{~min}$ with $35 \mathrm{kHz}$ [20]. The mixture is then placed in water bath at a temperature of $37^{\circ} \mathrm{C}$ for 1 hour. The process of ultra-sonication and maintaining temperature in the water bath is repeated 3 times. Precipitated products were collected and washed with ethanol several times. The filtrate is dried at $60^{\circ} \mathrm{C}$ for 1 hour [21]. 
Film Formation. The matrix used for film formation is cellulose acetate with cellulose microfibers and HA dispersed in it using CTAB as the surfactant [22-24]. Acetone is used as a solvent which evaporates when poured into the glass molds leaving behind thin flexible porous membranes. The ratios of the three can be varied according to the required flexibility and thickness of the membranes $[5,25]$.

\section{Results and Discussion}

X-Ray Diffraction. Curve Fig. 1(a) represents the XRD pattern of cellulose microfibers the peaks shown at 2 theta of $12^{\circ}, 20^{\circ}$ and $22^{\circ}$ are broad presenting the polymeric structure of cellulose microfibers that is it contains both amorphous and crystalline part [26]. The pattern is in accordance with the standard pattern found in reference library with an ICDD code of 00-003-0192. A little shift of peaks towards right that is, increased theta shows a decrease in d-spacing according to Bragg's law. The graph below in Fig. 1(b) represents the XRD pattern of treated and untreated raw cotton fibers. For comparison and to support our deduction it can be clearly seen that red graph representing treated fibers have sharper peaks with high intensity relative to that of untreated fibers. This suggests that after treatment the fibers become more crystalline and short therefore XRD peaks are sharper and intense with low full width half maxima [26].

Scanning Electron Microscopy. These images in Fig. 1 (c), (d), (e), (f) show the change in size and morphology of the raw cotton fibers after treatment in sulfuric acid. It can be seen from the images of scanning electron microscopy that the fiber length of cotton fibers is reduced by basic and acidic treatments which included sourcing, bleaching and acidic treatment $[4,11]$. The raw cotton fibers are larger in length comparatively and the surface of fibers obtained is smooth except some of the depositions visible on the images which are mainly due to the presence of hemi cellulose and lignin type impurities. The average length of fibers after treatments is 85 micron with a thickness of around 11 microns. On the other hand raw cotton fiber length is more than 500 micron and a diameter of around 25 microns. This difference clearly represents a size reduction in fiber length and diameter due to treatments thus producing cellulose micro fibers.

Fourier Transformation Infrared Spectroscopy. Curve in Fig. 2 confirms the presence of labeled bonds by determining frequency of particular bond and comparing it with standard frequency of those bonds in literature thus confirming the cellulose molecule.

SEM Images for Composite Membrane. Images in Fig. 3 represent the surface morphology and texture of the composite membranes formed. Pure cellulose acetate membranes are smooth and have a plain texture although at higher magnification of 5000x porosity is identified of around 2-4 microns as shown in Fig. 3(a) and 3(b). While the other membranes in Fig. 3(c) and 3(d) contains $\mathrm{CA}$ as matrix with cellulose microfibers and hydroxyapatite as reinforcement shows fibers dispersed in the matrix at a magnification of 500x and pore size in these membranes is around 1-2 microns and the thickness of membrane calculated using ImageJ software is in the range of 50-60 microns.

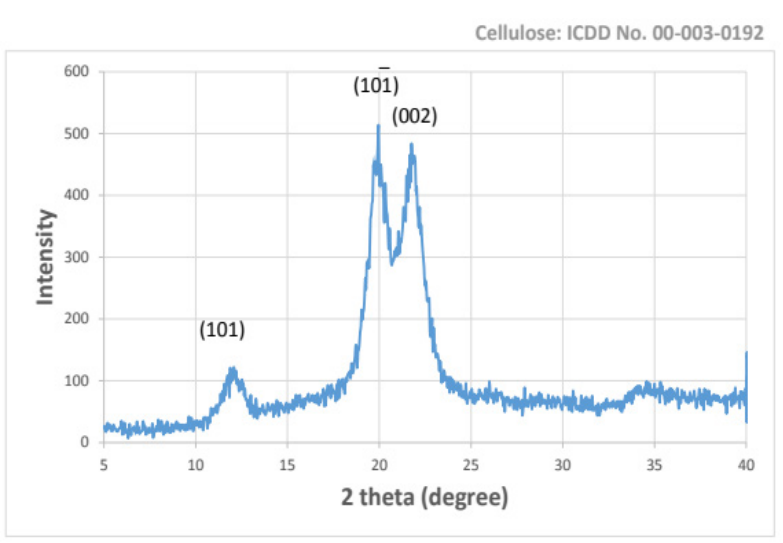

Fig. 1(a) XRD Curve for cellulose microfibers

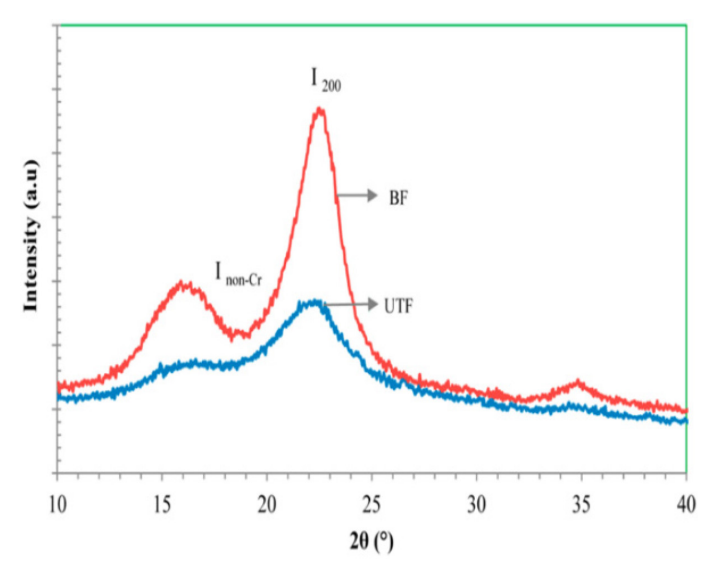

Fig. 1(b) XRD for CMF and cotton from literature 


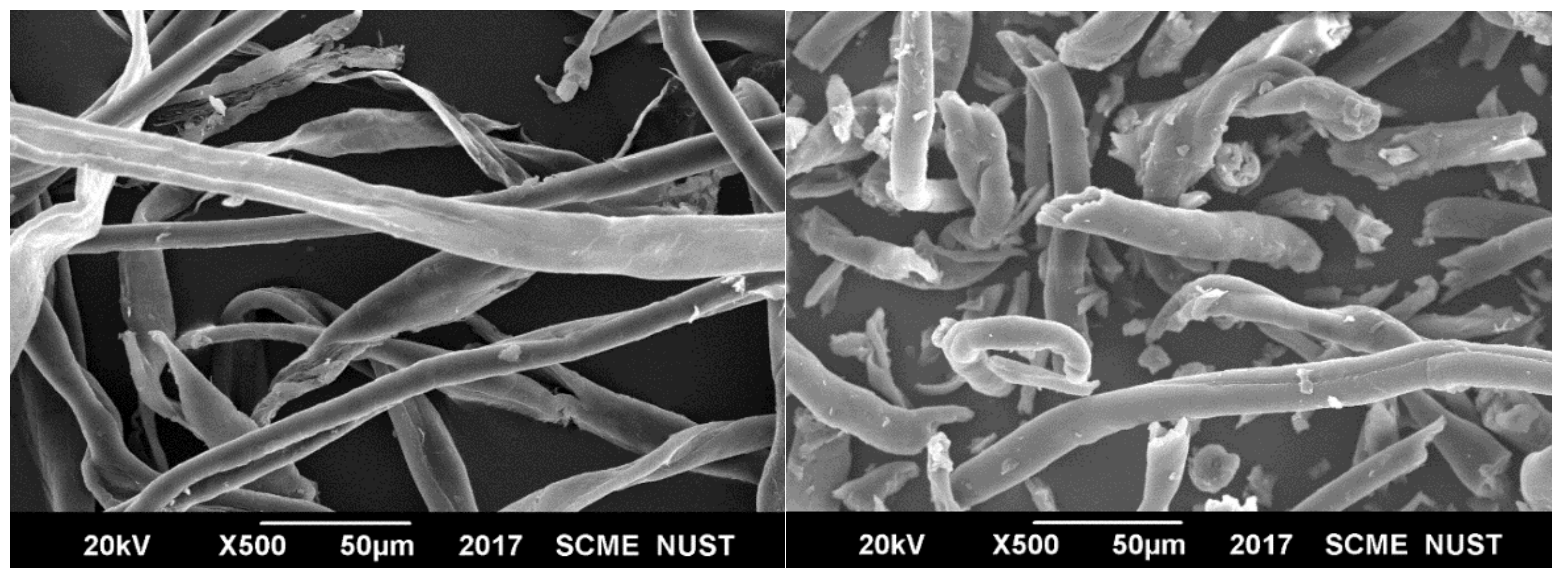

Fig. 1(c) SEM for raw cotton in fibrous form

Fig. 1(d) SEM for CMF at x500

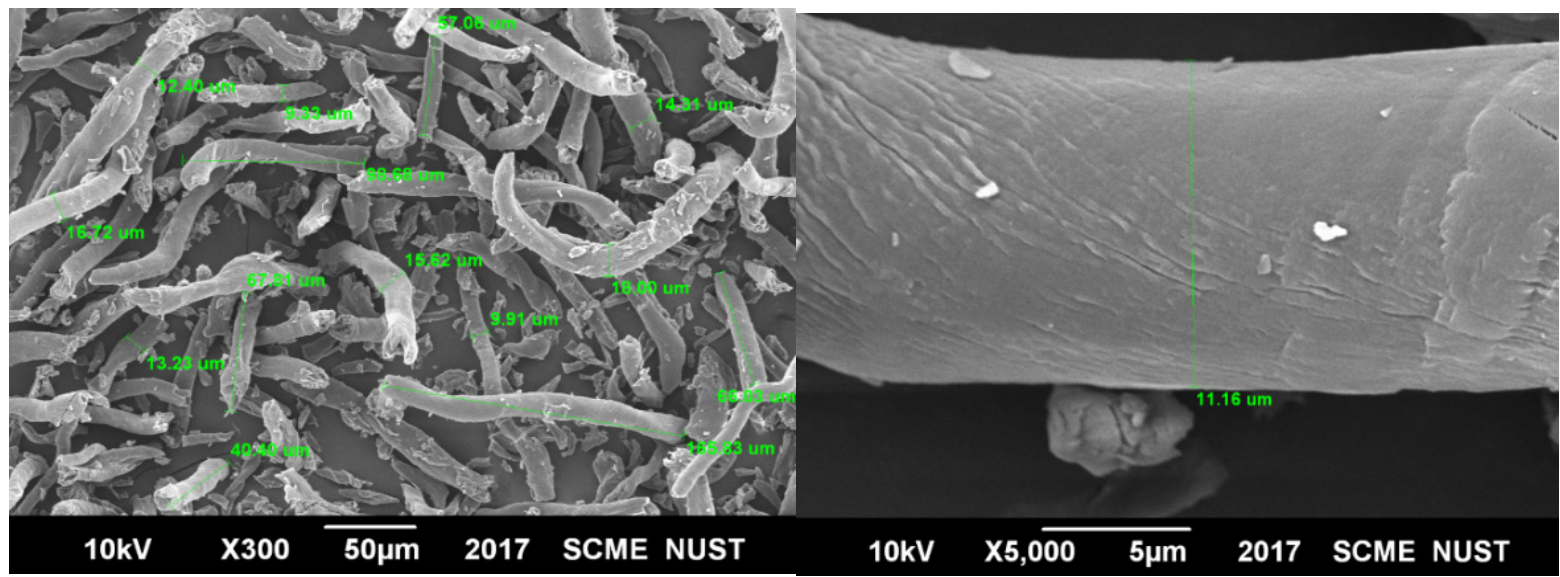

Fig. 1(e) SEM for CMF

Fig. 1(f) Surface of CMF

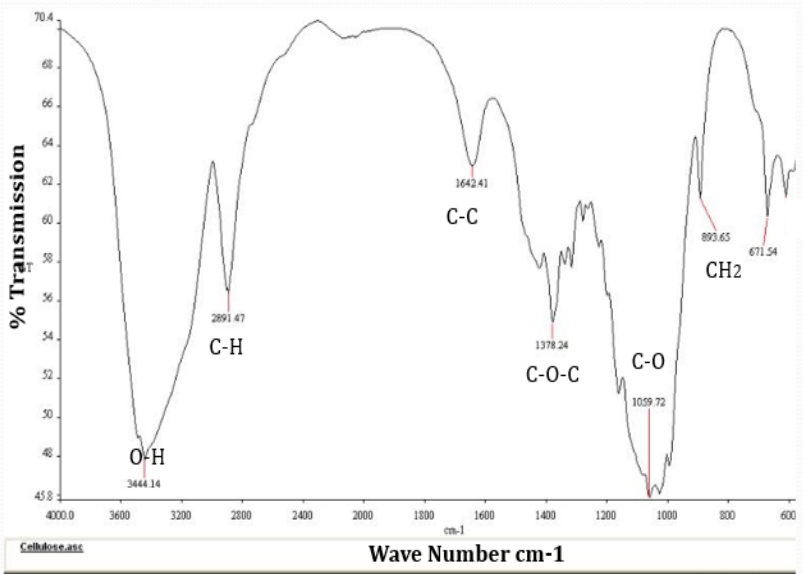

Fig. 2 FTIR of Cellulose Microfibers

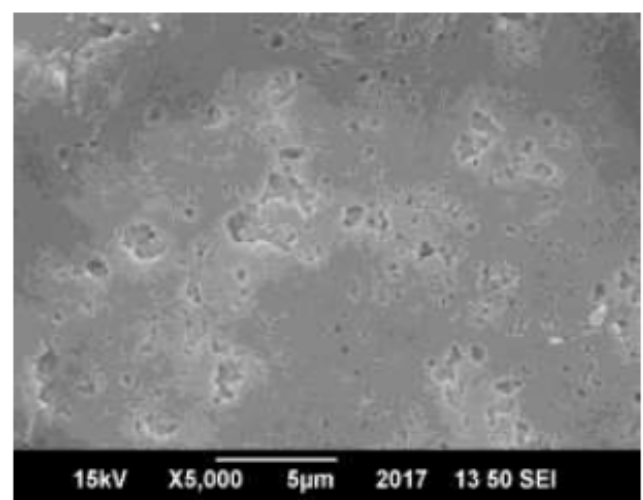

Fig. 3(a) Pure CA membrane surface

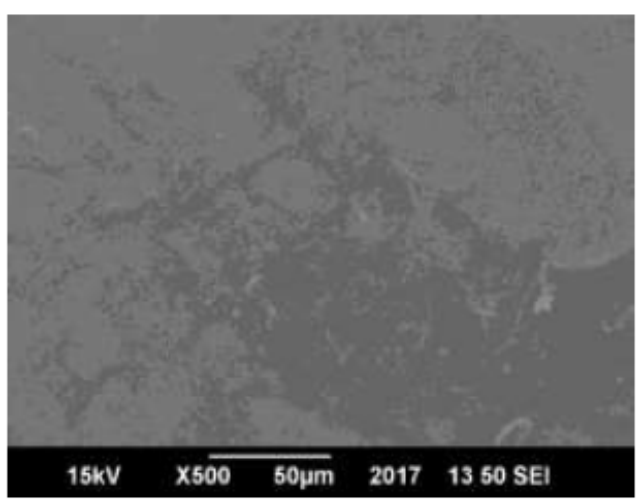

Fig. 3(b) Porous CA membrane surface 


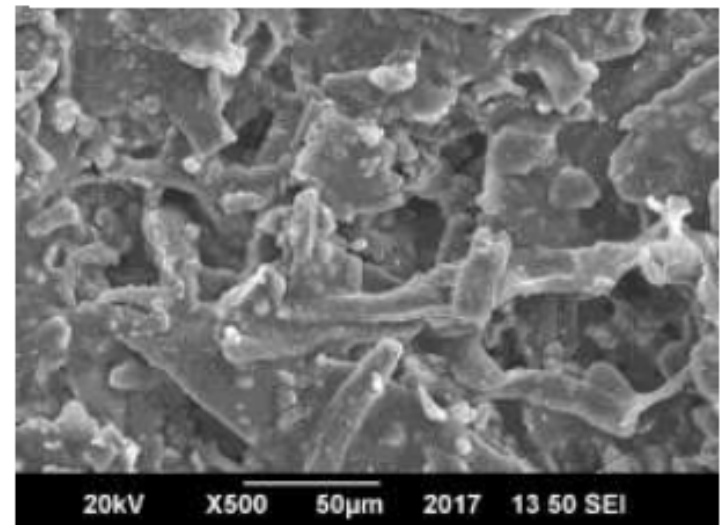

Fig. 3(c) Surface morphology

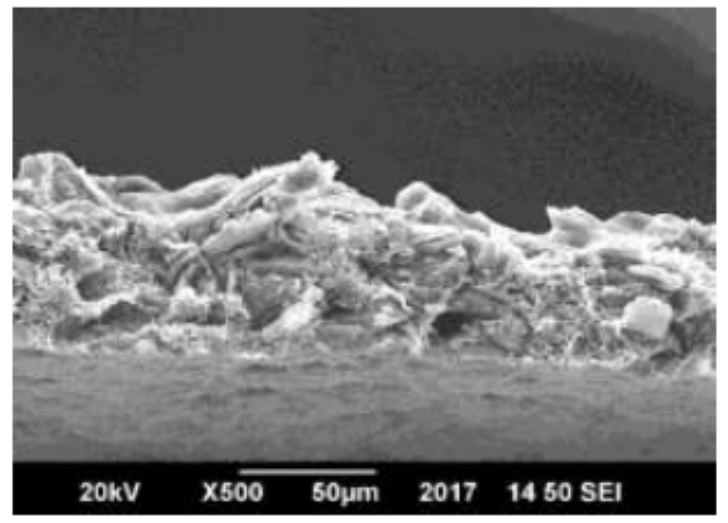

Fig. 3(d) Cross section of membrane

\section{Conclusions}

The membranes prepared using the following protocol is flexible enough to get rolled and be utilized in biomedical applications due to the already approved biocompatibility of the materials being used. The porosity achieved would also help in osteo-conductivity and thus bone regeneration. The membranes can also be further tested for gas and liquid permeation. The flexible sheets can also be rolled and stacked to increase the strength and enhance anisotropic properties.

\section{References}

[1] M. Ishikawa, Synthesis of Hydroxyapatite/Nanocellulose Composites, KTH Department of Fiber and Polymer Technology, Stockholm, Sweden, 2014.

[2] Z. Sheikh, S. Najeeb and Z. Khurshid, Biodegradable Materials for Bone Repair and Tissue Engineering Applications, Mater., 82015 5744-5794

[3] E.C. Moreno and T.M. Gregory, Preparation and Solubility of Hydroxyapatite, Research of the National Bureau of Standards, vol. 72A, 1968.

[4] H.C. Wu and T.W. Wang, Development and Characterization of Bioinspired Bone Matrix Aligned Nanocrystalline Hydroxyapatite on Collagen Nanofibers, J. Mat. Sci., 92016 3-5.

[5] M. Börjesson and G. Westman, Crystalline Nanocellulose - Preparation, Modification and Properties,Cellulose - Fundamental Aspects and Current Trends, Gothenburg, Sweden, 159-182, 2015.

[6] I. K. Ibrahim and S. M. Hussin, Extraction of Cellulose Nano Crystalline from Cotton by Ultrasonic and Its Morphological and Structural Characterization, Inter. J. Mat. Chem. Phy., 12015 99-109.

[7] K. Azzaouia and A. Lamhamdia, Synthesis and characterization of composite based on cellulose acetate and hydroxyapatite application to the absorption of harmful substances, Carbohydr. Polym., 24 2014 41-46.

[8] M. Zaborowska, A. Bodin and H. Bäckdahl, Microporous bacterial cellulose as a potential scaffold for bone regeneration, Act. Biomate., 62010 2540-2547.

[9] M. Lipowiecki and D. Brabazon, Design of Bone Scaffolds Structures for Rapid Prototyping with Increased Strength and Osteoconductivity, Advan. Mater. Res., 83-86 2010 914-922.

[10] C. J. Grande and F. G. Torres, Nanocomposites of bacterial cellulose/hydroxyapatite for biomedical applications, Act. Biomater., 52009 1605-1615. 
[11] Y. Wang, Cellulose Fiber Dissolution In Sodium Hydroxide Solution At Low Temperature: Dissolution Kinetics And Solubility Improvement,Georgia Institute of Technology, Georgia, USA, 2008.

[12] A. Boujemaoui and S. Mongkhontreerat, Preparation and characterization of functionalized cellulose nanocrystals, Carbohyd. Poly., 1152015 457-464.

[13] K. Ohkawa, Nanofibers of Cellulose and Its Derivatives Fabricated Using Direct Electrospinning, Molec., 202015 9139-9154

[14] C. J. Chirayil, L. Mathew and S. Thomas, Review Of Recent Research In Nano Cellulose Preparation From Different Lignocellulosic Fibers, Rev. Adv. Mater. Sci., 37 2010 20-28.

[15] M. Jorfi and E. J. Foster, Recent advances in nanocellulose for biomedical applications, Appl. Poly. Sci., 1322015 1-19.

[16] X. Lu and Y. Leng, Theoretical analysis of calcium phosphate precipitation in simulated body fluid, Biomat., 262005 1097-1108.

[17] A. Oyane, H. M. Kim and T. Furuya, Preparation and assessment of revised simulated body fluids, J Biomed Mater Res A, 652003 188-95.

[18] J. L. Varela Caselis, E. Reyes Cervantes, G. Landeta Cortés, R. Agustín Serrano, and E. Rubio Rosas, Hydroxyapatite growth on cotton fibers modified chemically, Mater. Sci.-Pol., 322014 436441.

[19] K. Yamaguchi and M. Prabakaran, Highly dispersed nanoscale hydroxyapatite on cellulose nanofibers for bone regeneration, Mater. Lett., 1682016 56-61.

[20] C. J. Malm and L. J. Tanghe, Chemical Reactions in the Making of Cellulose Acetate, Indust. Engineer, Chem., 471955 995-999.

[21] S. Saska, H. S. Barud and A. M. M. Gaspar, Bacterial Cellulose-Hydroxyapatite Nanocomposites for Bone Regeneration, Internat. J. Biomat., 152011 1-8.

[22] S. Park, J. O. Baker and M. E. Himmel, Cellulose crystallinity index: Measurement techniques and their impact on interpreting cellulase performance, Biotechn. Biofuels., 32010 1-1.

[23] Y. Tanga, X. Shen and J. Zhang, Extraction of cellulose nano-crystals from old corrugated container fiber using phosphoric acid and enzymatic hydrolysis followed by sonication, Carbohyd. Poly., 1252015 360-366.

[24] B. D Cullity and S.R Stock, Elements of X-Ray Diffraction, Addison-Wesley Publishing, pp. 258-293, 1956

[25] P. Echlin, Handbook of Sample Preparation for Scanning Electron Microscopy and X-Ray Microanalysis, Springer, 2011.

[26] B. C. Smith, Fundamentals of Fourier Transform Infrared Spectroscopy, CRC Press, 2011. 